\title{
Thalamic-Cortical-Striatal Circuitry Subserves Working Memory during Delayed Responding on a Radial Arm Maze
}

\author{
Stan B. Floresco, Deanna N. Braaksma, and Anthony G. Phillips \\ Department of Psychology, University of British Columbia, Vancouver, British Columbia, Canada V6T $1 Z 4$
}

The medial dorsal nuclei of the thalamus (MDNt), the prefrontal cortex, and the ventral striatum form an interconnected neural circuit that may subserve certain types of working memory. The present series of experiments investigated functional interactions between these brain regions in rats during the performance of delayed and nondelayed spatially cued radial-arm maze tasks. In Experiment 1, transient inactivation of the MDNt by a bilateral injection of lidocaine selectively disrupted performance on a delayed task but not on a nondelayed random foraging version of the radial arm maze task. In Experiment 2, asymmetrical lidocaine injections into the MDNt on one side of the brain and the prefrontal cortex on the other transiently disconnected these two brain regions and significantly impaired foraging during the delayed task. Similarly, disconnec- tions between the prefrontal cortex and the nucleus accumbens also disrupted foraging on this task, whereas disconnections between the MDNt and the nucleus accumbens had no effect. These data suggest that serial transmission of information among the MDNt, the prefrontal cortex, and the nucleus accumbens is required when trial-unique, short-term spatial memory is used to guide prospective search behavior. The results are discussed with respect to a distributed neural network linking limbic, thalamic, cortical, and striatal regions, which mediates executive functions of working memory.

Key words: medial-dorsal thalamus; prefrontal cortex; nucleus accumbens; working memory; neural networks; lidocaineinduced reversible lesions; rats
Working memory is defined as the ability to retain and manipulate mnemonic information to guide ongoing behavior (Baddeley, 1986). An important component of working memory is the shortterm storage of trial-unique information (Goldman-Rakic, 1995), whereby unique information about specific stimuli (e.g., spatial location and object information) is retained briefly in a short-term memory buffer and discarded after an appropriate response is executed. Another element of working memory involves cognitive processes that are characterized as "executive functions," which include the supervisory processes for the temporal organization of behavior, and the use of short-term memory to plan a sequence of forthcoming responses (Shallice, 1982; Baddeley and Della Sala, 1996; Shallice and Burgess, 1996; Kimberg et al., 1997).

There is a general consensus that the prefrontal cortex (PFC) is intimately involved in one or both aspects of working memory. Damage to the PFC in primates (Goldman-Rakic, 1987) or rats (Dunnett, 1990) impairs delayed responding on delayed response tasks with short delays. Likewise, electrophysiological recordings from PFC neurons in behaving primates (Fuster, 1995; GoldmanRakic, 1995) or rodents (Orlov et al., 1988; Batuev et al., 1990) show sustained firing throughout the delay period of a delayed response task, and this may serve as an internal representation of

\footnotetext{
Received Aug. 12, 1999; revised Oct. 5, 1999; accepted Oct. 7, 1999.

This research was supported by a grant from the Natural Sciences and Engineering Research Council of Canada to A.G.P. S.B.F is a recipient of a University Graduate Fellowship. We wish thank Sheheen Mithani and Kam Brar for their assistance with behavioral testing and Jeremy K. Seamans for useful discussions. Part of this paper were presented in abstract form at the 27th Annual Meeting of the Society for Neuroscience, San Diego, CA, 1997.

Correspondence should be addressed to Anthony G. Phillips, Department of Psychology, University of British Columbia, 2136 West Mall, Vancouver, BC, Canada V6T 1Z4. E-Mail: stanbf@unixg.ubc.ca.

Ms. Braaksma's present address: Dalhousie University, Halifax, Nova Scotia, Canada B3H $1 \mathrm{~J} 4$.

Copyright (C) 1999 Society for Neuroscience $0270-6474 / 99 / 1911061-11 \$ 05.00 / 0$
}

previously presented stimuli. Lesion and electrophysiological recording studies also have shown that the PFC mediates behaviors that require the manipulation of information for the purposes of executive motor control, including route planning in a Morris water maze (Granon and Poucet, 1995), switching of behavioral strategies (Seamans et al., 1995: Ragozzino et al., 1999), and the use of previously acquired information to guide prospective responding (Seamans et al., 1995; Rainer et al., 1999). Collectively, these studies suggest that the PFC plays an important role in both the short-term memory and executive components of working memory.

A growing body of evidence suggests that interactions between the PFC and its cortical and subcortical connections facilitate behaviors that require working memory. In primates, inactivation of either the parietal cortex (Quintana et al., 1989) or the inferotemporal cortex (Fuster et al., 1985) disrupts both behavioral performance and task-related neural activity in the PFC during a delayed matching-to-sample task, suggesting that information transfer between these brain regions meditates performance of this task. Similarly, disconnections between the temporal and frontal lobes disrupts learning of a conditional visual discrimination task (Gutnikov et al., 1997). Along similar lines, research in our laboratory has examined the interactions between the PFC and its limbic and striatal connections during the performance of a delayed spatial win-shift (SWSh) version of the radial arm maze task. During this task, rats are given information about the location of food on a maze during a training phase, $30 \mathrm{~min}$ before a test session. This procedure biases rats to forage prospectively (Cook et al., 1985), enabling them to plan a response strategy that ensures that food is located efficiently. Performance of this task is dependent, in part, on a neural circuit linking the hippocampus, PFC, and the nucleus accumbens (N.Acc.) (Floresco et al., 1997). We hypothesize that a distributed neural network involving the 
frontal and temporal corticies mediates the retrieval and use of trial-unique spatial information over extended delays, whereas a circuit linking the PFC to the ventral striatum is involved in integrating a prospective code into appropriate motor output.

The PFC is also linked anatomically to the medial dorsal nuclei of the thalamus (MDNt). The MDNt shares reciprocal connections with the PFC (Krettek and Price, 1977; Leonard, 1969; Ray and Price, 1992) and the hippocampal formation (Beckstead, 1978; Su and Bentivoglio, 1990) and also sends projections to the N.Acc. (Berendse and Groenewegen, 1990; Otake and Nakamura, 1998). The distributed interconnectivity of the MDNt suggests that this region of the thalamus may play an important role in different components of working memory subserved by these other brain regions. This conjecture is supported by lesion and electrophysiological studies implicating the MDNt in a broad range of cognitive processes, including object recognition (Mumby et al., 1993), short-term memory (Harrison and Mair, 1996), planning and prospective coding (Joyce and Robbins, 1991; Gallassi et al., 1992; Daum and Ackermann, 1994), strategy selection and behavioral flexibility (Hunt and Aggleton, 1998), the encoding of the motivational significance of stimuli (Gabriel, 1993; Oyoshi et al., 1996), and working memory (Freeman et al., 1996; Callicott et al., 1999).

The present study investigated the role of the MDNt during performance of two variants of the radial arm maze task in rats, each of which required a different type of memory processing, one involving retrospective and the other prospective use of previously acquired information. The first experiment assessed the effects of bilateral reversible lidocaine inactivation of the MDNt before either the training or the test phase of the delayed SWSh task, or the nondelayed random foraging (RF) version of the radial maze task. The second experiment used asymmetrical disconnection lesions (Floresco et al., 1997) among the MDNt, the PFC, and the N.Acc. to elucidate the routes of information transfer in thalamic-cortical-striatal circuits essential for performance of the delayed SWSh task

\section{MATERIALS AND METHODS}

\section{Subjects}

The subjects were male Long-Evans rats weighing between 300 and 450 gm before surgery. All rats were given ad libitum access to water and were maintained at $85 \%$ of their free-feeding weight by providing $25-30$ gm of Purina lab chow pellets once daily. Rats were tested 5-7 d/week.

\section{Surgery}

Rats were anesthetized with $100 \mathrm{mg} / \mathrm{kg}$ ketamine hydrochloride and 7 $\mathrm{mg} / \mathrm{kg}$ xylazine. Twenty-three gauge stainless-steel guide cannulae were implanted into the brain regions using standard stereotaxic techniques. The stereotaxic coordinates (flat skull) were derived from those of Paxinos and Watson (1986). For the bilateral MDNt inactivation experiments (Experiment 1), three groups of rats were implanted with one set of bilateral cannula into the MDNt [anteroposterior (AP), $-2.9 \mathrm{~mm}$ from bregma; mediolateral (ML), $\pm 0.7 \mathrm{~mm}$ from midline; and dorsoventral (DV), $-4.9 \mathrm{~mm}$ from dura].

For the disconnection experiments (Experiment 2), three groups of rats were implanted with two sets of bilateral cannula. One group of rats was implanted with one pair of cannula in the MDNt and a second pair in the prelimbic region of the PFC (AP, $+2.7 \mathrm{~mm}$; ML, $\pm 0.7 \mathrm{~mm}$ from bregma; and DV, $-3.0 \mathrm{~mm}$ from dura). This region of the PFC was chosen because it receives projections from the hippocampus (Conde et al., 1995), and because it is involved selectively in delayed radial maze foraging (Seamans et al., 1995; Floresco et al., 1997). A second group was implanted with one pair of cannula in the MDNt and a second pair in the N.Acc. (AP, $+1.5 \mathrm{~mm}$; ML, $\pm 1.3 \mathrm{~mm}$ from bregma; and DV, $-6.0 \mathrm{~mm}$ from dura). A third group of rats was implanted with one pair of cannula in the PFC and a second pair in the N.Acc. In this group, the corpus callosum was transected with a 30 gauge needle (AP, +1.6 to $+0.5 \mathrm{~mm}$; $\mathrm{ML}, \pm 1.0 \mathrm{~mm}$ from bregma; and $\mathrm{DV},-4.0 \mathrm{~mm}$ from dura). This procedure ensured that there would be no projections from one hemisphere of the PFC that would reach the contralateral N.Acc. (Brog et al., 1993). Unilateral infusions of lidocaine were administered in the hemisphere opposite to the side that received the transection. Thirty gauge obdurators flush with the end of the guide cannulae remained in place until the injections were made. Each rat was given at least $7 \mathrm{~d}$ to recover from surgery before testing.

\section{Microinfusion procedure}

On injection days, the obdurators were removed, and 30 gauge stainless steel injection cannulae were inserted $0.8 \mathrm{~mm}$ beyond the tip of the guide cannulae. Lidocaine ( $20 \mu \mathrm{g}$ in $0.5 \mu \mathrm{l}$ of saline; Astra Pharmaceuticals and Research Biochemicals) or saline $(0.5 \mu \mathrm{l})$ were delivered at a rate of 0.5 $\mu 1 / 1.2$ min by a microsyringe pump (Sage Instruments model 341). Injection cannulae were left in place for an additional 1 min after each injection to allow for diff usion. Each rat remained in its home cage for an additional $3 \mathrm{~min}$ before being placed on the maze. The functional spread of lidocaine infused at this volume and concentration has been estimated previously at no greater than $1 \mathrm{~mm}$ in diameter (Floresco et al., 1997; Tehovnik and Sommer, 1997). Based on these estimates, it could be expected that the lidocaine infusions in the present study would inactivate the medial, central, and lateral regions of the medial dorsal thalamus, as well as the paraventricular nuclei.

\section{Apparatus}

An eight-arm radial maze was used for all experiments. The maze had an octagonal center platform $40 \mathrm{~cm}$ in diameter connected to eight equally spaced arms, each measuring $50 \times 9 \mathrm{~cm}$, with a cylindrical food cup at the end. Removable pieces of white opaque plastic $(9 \times 13 \mathrm{~cm})$ were used to block the arms of the maze. The maze was elevated $40 \mathrm{~cm}$ from the floor and was surrounded by numerous extra maze cues (e.g., cupboards, posters, doors, and the experimenter), in a room $4 \times 5 \times 3 \mathrm{~m}$, which was illuminated with overhead fluorescent lights $(100 \mathrm{~W})$.

\section{Foraging tasks}

The two foraging tasks used in the present study were the delayed SWSh and the nondelayed RF tasks.

The delayed SWSh task. This task was adapted from that of Packard et al. (1990) and has been described in detail elsewhere (Floresco et al., 1997). On the first $2 \mathrm{~d}$ of testing, rats were habituated to the maze environment. Subsequent training trials were given once daily. These trials consisted of a training phase and a test phase, separated by a delay. Before the training phase, a set of four arms was chosen randomly and blocked. Food pellets (Bioserv, Frenchtown, NJ) were placed in the food cups of the four remaining open arms. During the training phase, each rat was given 5 min to retrieve the pellets from the four open arms and then was returned to its home cage for the delay period (see below). During the test phase of each daily trial, all arms were open, but only the arms that were previously blocked contained food. Rats were allowed a maximum of 5 min to retrieve the four pellets during the test phase.

The initial delay between training and test phases was 5 min. After achieving criterion performance in which all four pellets were retrieved in five or fewer choices during the test phase for two consecutive days, the delay was increased to $30 \mathrm{~min}$. The first intracranial injections were administered after rats attained 2 consecutive days of criterion performance at a 30 min delay. After the first injection day, animals were again retrained to the criterion performance. The following day, a second intracranial injection was administered. This procedure was repeated until an animal had received the designated sequence of injections, according to the protocols described below (see Procedure).

On injection days the number and order of arm entries were recorded. An arm entry was recorded when a rat moved down the entire length of an arm and reached the food cup at the end of the arm. Errors were scored as entries into nonbaited arms and further broken down into two error subtypes. An across-phase error was defined as any initial entry into an arm that had been visited previously during the training phase. A within-phase error was any reentry into an arm that had been entered earlier during the test phase. The latencies to reach the food cup of the first arm visited and to complete the phase also were recorded.

The nondelayed RF task. This task also has been described elsewhere (Floresco et al., 1997). Habituation to the maze during the first $2 \mathrm{~d}$ of training was identical to the delayed SWSh procedure described above. On subsequent daily trials animals were required to forage for pellets 
placed at random in the food cups of four of the eight arms (hence the term random foraging). A novel set of arms was baited each day. Animals were trained to a criterion of no more than one reentry error per trial for 4 consecutive days. The day after criterion performance was achieved, the first intracranial injections were administered. After the first injection day, animals were retrained to criterion for 2 consecutive days. As with the delayed SWSh task, this procedure was repeated until each animal had received all the designated injections.

Errors were scored as reentries into arms visited previously within a trial. These errors were broken down further into reentries into baited arms (arms that had been baited at the start of the trial) and reentries to nonbaited arms (arms that were not baited before the start of the trial). The number of reentry errors made on each of the injection days was recorded and used for data analysis. As with the delayed SWSh paradigm, the latencies to reach the first food cup (either baited or nonbaited) after being placed on the maze and the time required to retrieve all four pellets were also recorded.

\section{Histology}

After completion of behavioral testing, the rats were killed in a carbon dioxide chamber. Brains were removed and fixed in a $10 \%$ formalin solution. The brains were frozen and sliced in $50 \mu \mathrm{m}$ sections before being mounted and stained with cresyl violet. Placements were verified with reference to the neuroanatomical atlas of Paxinos and Watson (1986).

\section{Data analysis}

The number and type of errors made on the day before the first injection sequence and for all injection days for each experiment were analyzed using separate two-way within-subjects design ANOVA with the treatment and error type as two within-subjects factors. In this design, a significant main effect of treatment indicates that one or more infusion treaments caused an increase in the total number of errors observed during the task, releative to other treatments. A significant main effect of error type would indicate that rats made significantly more of one type of error (e.g., across-phase errors) versus the other type of error (e.g., within-phase) over all treatment conditions. The $n$ values listed for each group represent the number of rats that had acceptable cannulae placements. Main effects of Treatment were further analyzed using Tukey's post hoc tests for repeated measures. Whenever a significant main effect of treatment was observed, one planned comparison was made, analyzing the number of each type of error made on lidocaine injection days.

The latency data to reach the first food cup and the average time per subsequent choice on injection days were analyzed with a one-way repeated measures ANOVA. The average time per subsequent choice was calculated using the formula: (time to complete trial - time to initiate trial) $\div$ number of choices for trial.

Whenever a significant main effect of treatment was observed in Experiment 2, three one-way ANOVAs were conducted, assessing the number of errors made on tests after a unilateral inactivation of the MDNt, PFC, or N.Acc., as well as after disconnection inactivations, with the side of the injection as a between-subjects factor. This analysis was conducted to rule out the possibility that unilateral inactivations in one hemisphere would lead to a greater increase in errors than inactivations of the other hemisphere.

\section{EXPERIMENT 1: REVERSIBLE, BILATERAL MDNT LESIONS}

\section{Procedure}

A within-subjects design was used for all three parts of Experiment 1. Three groups of rats with bilateral cannulae implanted into the MDNt were trained on either the delayed SWSh task or the RF task. On the first injection days, these three groups of rats received bilateral infusions of either lidocaine or saline into the MDNt before the nondelayed RF task (group 1), before the training phase (group 2), or before the test phase of the delayed SWSh (group 3). Animals were subsequently retrained until they reattained criterion performance. On the day after the criterion was reattained, a second infusion of either saline or lidocaine was administered in a counterbalanced order.

\section{Results}

\section{Nondelayed RF}

Before a daily trial of the nondelayed RF task, 11 rats received bilateral infusions of lidocaine or saline into the MDNt on sep- arate days. The analysis of these data revealed no significant main effect of treatment $\left(F_{(2,20)}=1.12\right.$; not significant; Fig. $\left.1 A\right)$. There were also no significant differences between treatment days in the latencies to reach the first food cup or on the average time per subsequent choice (all $F<0.55$; not significant).

\section{Delayed SWSh, pretraining injections}

Before the training phase of the delayed SWSh task, eight rats received bilateral infusions of lidocaine or saline into the MDNt on separate days. There were no significant differences in the number of errors made on either the training phase $\left(F_{(2,12)}=\right.$ 1.00; not significant; Fig. $1 B$, inset $)$ or the test phase $\left(F_{(2,12)}=\right.$ 1.28; not significant; Fig. $1 B$ ) on any of the treatment days. In addition, there were no significant differences in latencies to reach the first food cup or in the average time per subsequent choice of arms on either the training or the test phases (all $F<2.3$; not significant).

\section{Delayed SWSh, pretest injections}

Before the test phase of the delayed SWSh task, eight rats received bilateral infusions of lidocaine or saline into the MDNt on separate days. Analyses of the number of errors made on the day before the first injection and on saline and lidocaine injection days revealed a significant main effect of treatment $\left(F_{(2,12)}=24.08\right.$; $p<0.001$; Fig. 1C). Tukey's post hoc analysis for repeated measures showed that rats made significantly more errors on lidocaine injection days relative to both the day before the first injection and the saline injection days $(p<0.001)$. Subsequent planned comparisons on the type of errors made on lidocaine injection days revealed that rats made the same number of acrossphase and within-phase errors $\left(F_{(1,7)}=0.07\right.$; not significant; Fig. $1 C$, inset). Furthermore, there were no significant differences between injection days on the latencies to reach the first food cup $\left(F_{(2,14)}=1.31\right.$; not significant.). However, lidocaine inf usions into the MDNt did increase the average time per subsequent choice (mean, $19.38 \mathrm{sec}$ ) relative to saline treatments and the day before the first injection (mean, $13.48 \mathrm{sec} ; F_{(2,14)}=3.80 ; p<0.05$; and Tukey's $p<0.05)$.

\section{Histology}

The locations of the inf usion sites for all rats receiving inactivation of the MDNt before the test phase of the delayed SWSh task are shown in Figure $1 D$. Rats whose inf usions were asymmetrical in the dorsoventral plane or whose placements encroached on either the hippocampus or were near the region of the mammillothalamic tract-gelatinosus nucleus were not included in the analysis $(n=5)$. It is notable that rats whose placements encroached on the hippocampus tended to be impaired on the tasks, and those rats with placements near the level of the mammillothalamic tract showed no discernible impairment on any of the tasks. The data from these rats were not included in the analyses.

\section{Discussion}

Reversible lesions of the MDNt selectively disrupted performance of the delayed SWSh task only when administered before the test phase. Lidocaine infusions had no effect on performance when they were administered before the training phase of the delayed SWSh task or before the nondelayed RF task. The data from this experiment suggest that the MDNt does not play a significant role in either the acquisition of trial-unique information or in exploratory goal-directed locomotion in the absence of previous knowledge about the location of food. Rather, these data 
Figure 1. Effects of bilateral inactivation of the MDNt on performance of delayed and nondelayed radial arm maze tasks. $A$, Number of errors (mean \pm SEM) made by rats on the day before the first injection (open bar) and after infusions of saline (hatched bar) or lidocaine (black bar) into the MDNt before the nondelayed RF task. $B$, Number of errors (mean \pm SEM) made by rats during the test phase on the day before the first injection (open bar) and after infusions of saline (hatched bar) or lidocaine (black bar) into the MDNt before the training phase of the delayed SWSh task. Inset, Number of errors made during the training phase on the day before the first injection (open bar) and on saline (hatched bar) and lidocaine (black bar) injection days. $C$, Number of errors (mean \pm SEM) made by rats during the test phase on the day before the first injection (open bar) and after infusions of saline (hatched bar) or lidocaine (black bar) into the MDNt before the test phase of the delayed SWSh task. ${ }^{\star \star}$ Significance at $p<0.001$ relative to saline and previous day. Inset, Number of across-phase errors (cross-hatched bar) and within-phase errors (striped bar) made by rats on lidocaine injection days. $D$, Location of infusions (black circles) for all rats with acceptable placements receiving infusions into the MDNt before the test phase of the delayed SWSh task. Plates are computer-generated adaptations from Paxinos and Watson (1986). Numbers beside each slide correspond to millimeters from bregma.

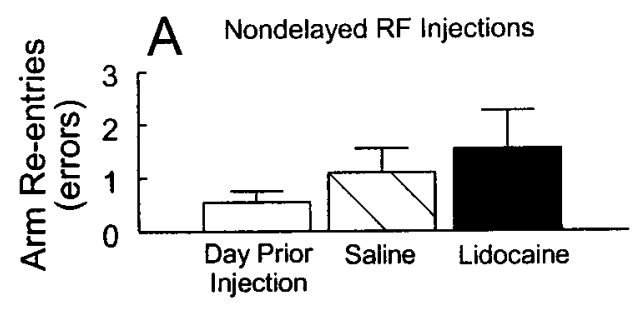

B Delayed SWSh, Pretraining Injections

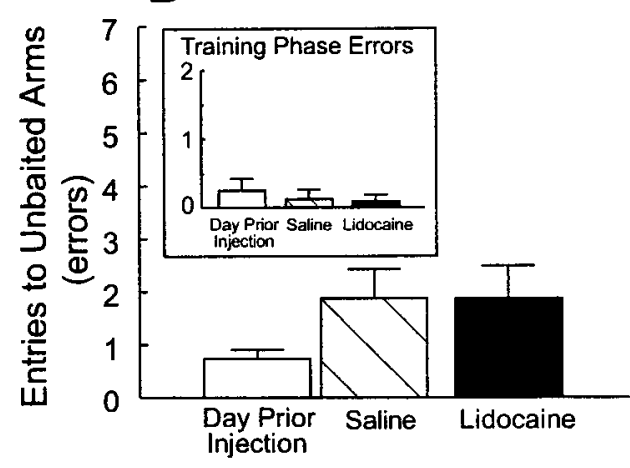

C Delayed SWSh, Pretest Injections

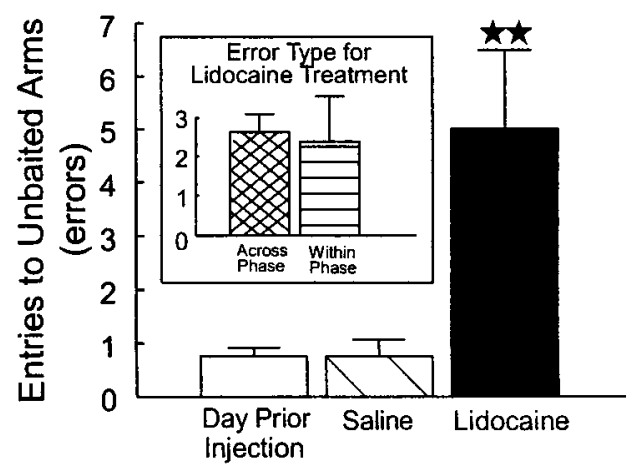

\section{Locations of Infusions}

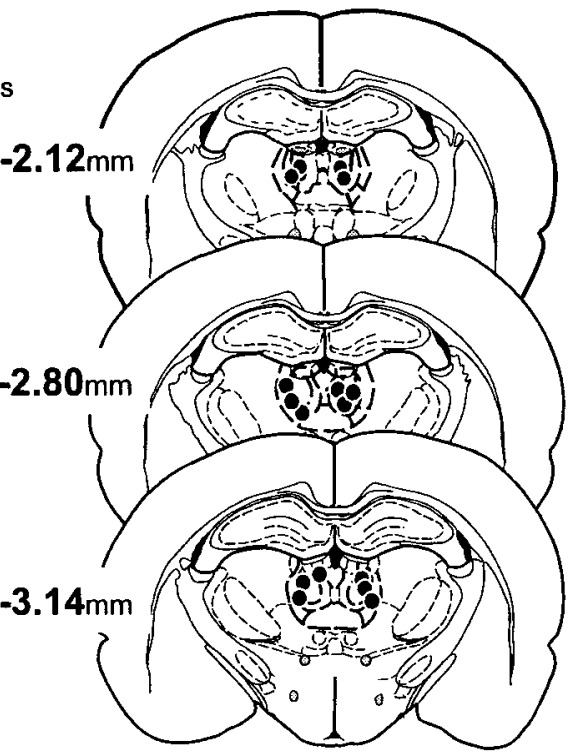

support the conclusion that the MDNt plays a specific role in the retrieval and use of previously acquired information to guide ongoing behaviors after a delay. As such, the present data are consistent with previous reports that lesions to the rat MDNt do not impair performance of nondelayed radial arm maze tasks (Kessler et al., 1982; Kolb et al., 1982; but see Stokes and Best, 1990) but do disrupt the performance of radial maze tasks with delays between choices (Kessler et al., 1982; Harrison and Mair, 1996; Mair et al., 1998).

Analysis of the type of errors made by rats after reversible lesions of the MDNt was particularly revealing. During the delayed SWSh task, rats made an equal number of errors to arms entered $30 \mathrm{~min}$ previously during the training phase (across-phase errors) and those entered more recently during the test phase (within-phase errors; Fig. 1B, inset). This finding is consistent with other studies demonstrating that lesions of the MDNt result in similar impairments on a variety of delayed memory tasks (Winocur, 1985; Mumby et al., 1993; Savage et al., 1997; Mair et al., 1998). It is interesting to note that the pattern of deficits after inactivations of the MDNt was identical to those observed after similar inactivations of the prelimbic cortex of the PFC (Seamans et al., 1995), or after disconnections between the PFC and the hippocampus (Floresco et al., 1997). In those studies, the observation of an equal number of across- and within-phase errors on the delayed SWSh task, in combination with no impairment on the nondelayed RF task, was interpreted as a disruption in the ability to use previously acquired information to guide behavior toward arms predictive of food reward, as opposed to an impairment in short-term memory processes. A similar explanation may follow for the selective impairment in delayed SWSh behavior after reversible lesions of the MDNt. Inactivations of the MDNt may have disrupted the ability of rats to use information acquired during the training phase to discriminate which arms would contain food during the test phase.

Insight into the exact role played by the MDNt in delayed SWSh behavior may be obtained by examining interactions with its efferent structures. As mentioned previously, the MDNt is reciprocally connected with the PFC (Leonard, 1969; Krettek and Price, 1977; Ray and Price, 1992) and also sends projections to the N.Acc. (Berendse and Groenewegen, 1990; Otake and Nakamura, 1998). It is notable that bilateral inactivations to either of these structures result in impairment on delayed SWSh performance similar to those observed here after MDNt inactivations (Seamans and Phillips, 1994; Seamans et al., 1995). Therefore, the MDNt may interact with the PFC and/or the N.Acc. when rats are engaged in prospective foraging behavior on the delayed SWSh task. Experiment 2 tested this hypothesis by using asymmetrical disconnection lesions between the MDNt and either the PFC or 


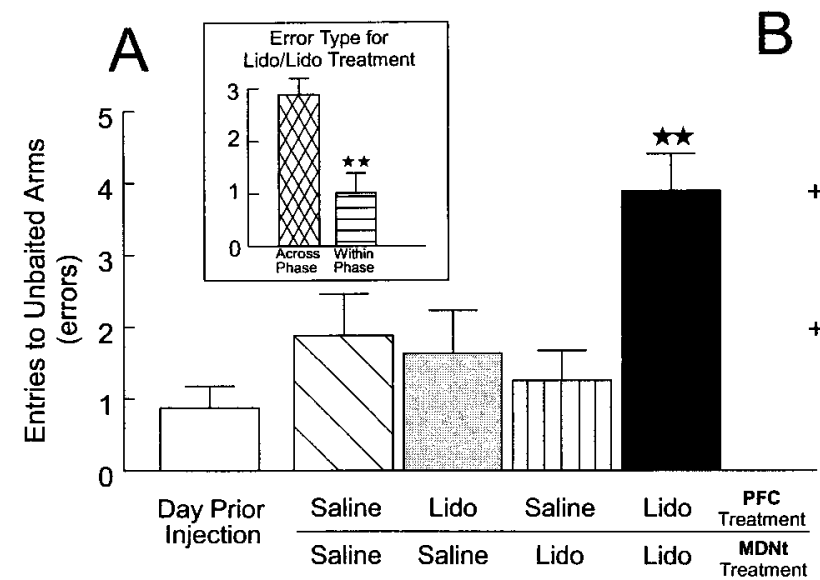

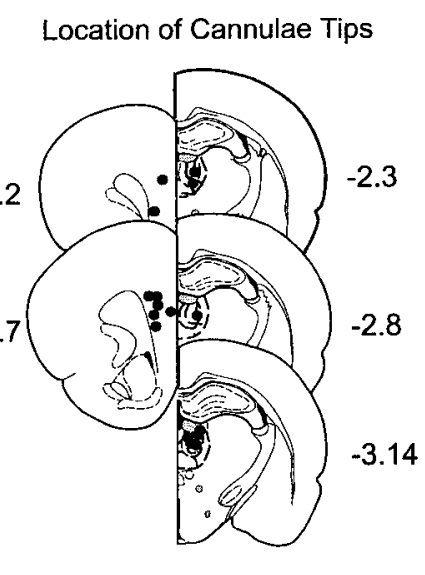

Figure 2. Effects of PFC-MDNt disconnections on performance of the delayed SWSh task. $A$, Number of errors (mean \pm SEM) made by rats on the day before the first injection (open bar) and after unilateral infusions of saline into both the PFC and the MDNt (hatched bar), unilateral infusions of lidocaine (Lido) into the PFC and contralateral saline in the MDNt ( graybar), unilateral infusions of Lido into the MDNt and contralateral saline into the PFC (stripped bar), and unilateral Lido into the MDNt and contralateral Lido into the PFC (disconnection; black bar) before the test phase of the delayed SWSh task. ${ }^{\star \star}$ Significance at $p<0.001$ versus all other treatment conditions. Inset, Number of acrossphase (cross-hatched bar) versus withinphase (striped bar) errors made by rats

during Lido/Lido (disconnection) injection days. ${ }^{\star \star S i g n i f i c a n c e ~ a t ~} p<0.001$ across- versus within-phase errors. B, Location of cannulae tips (black circles) for all rats used for data analysis receiving PFC-MDNt disconnections before the delayed SWSh task. Plates are computer-generated adaptations from Paxinos and Watson (1986). Numbers beside each plate correspond to millimeters from bregma. For clarity, $B$ and all subsequent histology figures represent the locations of cannulae tips on sides that received infusions on disconnection injection days. All animals received inf usions of either lidocaine or saline in each hemisphere.

the N.Acc. before the test phase of the delayed SWSh task. In addition, the PFC also sends a dense projection to the N.Acc. (Sesack et al., 1989; Brog et al., 1993), and it has been proposed that this corticostriatal pathway is responsible for the transformation of mnemonic information into a sequence of goal-directed motor responses (Robbins, 1990, 1991; Goldman-Rakic et al., 1992; Seamans et al., 1995; Floresco et al., 1997). Thus it was of interest to assess the effect of disconnections between the PFC and the N.Acc. on performance of the delayed SWSh task. A disruption in foraging behavior after asymmetrical lesions between the PFC and the N.Acc. would confirm the importance of this corticostriatal circuit in the transformation of a prospective plan of action into efficient behavioral output.

\section{EXPERIMENT 2: DISCONNECTION LESIONS BETWEEN THE MDNt AND THE PFC, THE MDNt AND THE N.Acc, OR THE PFC AND THE N.ACC}

\section{Procedure}

A within-subjects design was used for all three lesion conditions of Experiment 2. Three groups of rats were implanted with two sets of bilateral cannulae in the following combinations: MDNt-PFC (group 1), MDNt-N.Acc. (group 2), and PFC-N.Acc. (group 3). After recovery from surgery they were trained to criterion on the delayed SWSh task.

After achievement of criterion performance, animals in each of the disconnection lesion experiments received injections on 4 separate days, each before the test phase of the delayed SWSh task. The following combinations of asymmetrical bilateral infusions were used for groups 1 and 2 (MDNt-PFC and MDNt-N.Acc.): (1) a unilateral lidocaine infusion into the MDNt in combination with a contralateral lidocaine infusion into either the PFC or the N.Acc. (disconnection); (2) a unilateral lidocaine infusion into the MDNt in combination with a saline infusion into the contralateral PFC or N.Acc.; (3) a unilateral lidocaine inf usion into the PFC or N.Acc. in combination with a saline infusion into the contralateral MDNt; and (4) unilateral infusions of saline into the MDNt and saline infusions into either the contralateral PFC or the N.Acc. For group 3 (PFC-N.Acc.), the following combinations of asymmetrical bilateral infusions were used: (1) a unilateral lidocaine infusion into the PFC in combination with a contralateral lidocaine infusion the N.Acc. (disconnection); (2) a unilateral lidocaine infusion into the PFC in combination with a saline infusion into the contralateral N.Acc.; (3) a unilateral lidocaine infusion into the PFC in combination with a saline infusion into the contralateral N.Acc.; and (4) unilateral infusions of saline into both PFC and contralateral N.Acc. The order of infusions was counterbalanced between animals using a quasi-Latin square design. The counterbalancing was designed to ensure that a given sequence of injections was not repeated. The hemisphere (left or right) used for the first injection was also counterbalanced and was alternated for subsequent injections. Each animal was tested separately on the delayed SWSh task, and the injection procedure was repeated until the animal had been tested four times with each sequence of intracranial injections.

\section{Results}

\section{PFC-MDNt disconnections}

Rats $(n=8)$ with two sets of bilateral cannulae implanted into the prelimbic region of the PFC and the MDNt received the injection protocol described above before the test phase of the delayed SWSh task on 4 separate days. An analysis of these data revealed a significant main effect of treatment $\left(F_{(4,28)}=6.41 ; p<0.001\right)$. Tukey's post hoc test for repeated measures revealed that rats made significantly more errors on injection days on which the PFC and the MDNt both received lidocaine infusions and were disconnected versus all other injections days ( $p<0.01$; Fig. $2 A)$. There were no other significant differences in the number of errors made on any of the other injection days. There was a significant main effect of error type $\left(F_{(1,7)}=25,95 ; p<0.001\right)$. Subsequent planned comparisons on the type of errors made after PFC-MDNt disconnections showed that rats made significantly more across-phase versus within-phase errors $\left(F_{(1,7)}=\right.$ $18.1 ; p<0.01$; Fig. $2 A$, inset $)$. There was no significant treatment $\times$ error type interaction $\left(F_{(4,28)}=2.30\right.$; not significant $)$. A separate series of tests was conducted to assess any hemispheric biases on the number of errors made after unilateral PFC inactivations, unilateral MDNt inactivations, and the disconnection lesions. The analysis revealed no significant effects of the side of the injection on the number of errors made by rats on the three injection days (all $F<1.1$; not significant).

Histology. The location of cannulae tips for all rats receiving PFC-MDNt disconnections are represented in Figure 2B. Bilateral placements in the MDNt were similar to those observed in Experiment 1. Placements in the PFC were limited to the prelimbic cortex, similar to placements observed by Seamans et al. (1995).

\section{MDNt-N.Acc. disconnections}

A separate group of rats $(n=7)$ with two sets of bilateral cannulae implanted into the N.Acc. and the MDNt received the injection protocol described above before the test phase of the 
Figure 3. Effects of MDNt-N.Acc. disconnections on performance of the delayed SWSh task. $A$, Number of errors (mean \pm SEM) made by rats on the day before the first injection (open bar) and after unilateral infusions of saline into both the N.Acc. and the MDNt (hatched bar), unilateral infusions of lidocaine (Lido) into the N.Acc. and contralateral saline in the MDNt ( gray bar), unilateral inf usions of Lido into the MDNt and contralateral saline into the N.Acc. (striped bar), and unilateral Lido into the MDNt and contralateral Lido into the N.Acc. (disconnection; black bar) before the test phase of the delayed SWSh task. $B$, Location of cannulae tips (black circles) for all rats used for data analysis receiving MDNt-N.Acc. disconnections before the delayed SWSh task. Plates are computer-generated adaptations from Paxinos and Watson (1986). Numbers beside each plate correspond to millimeters from bregma.
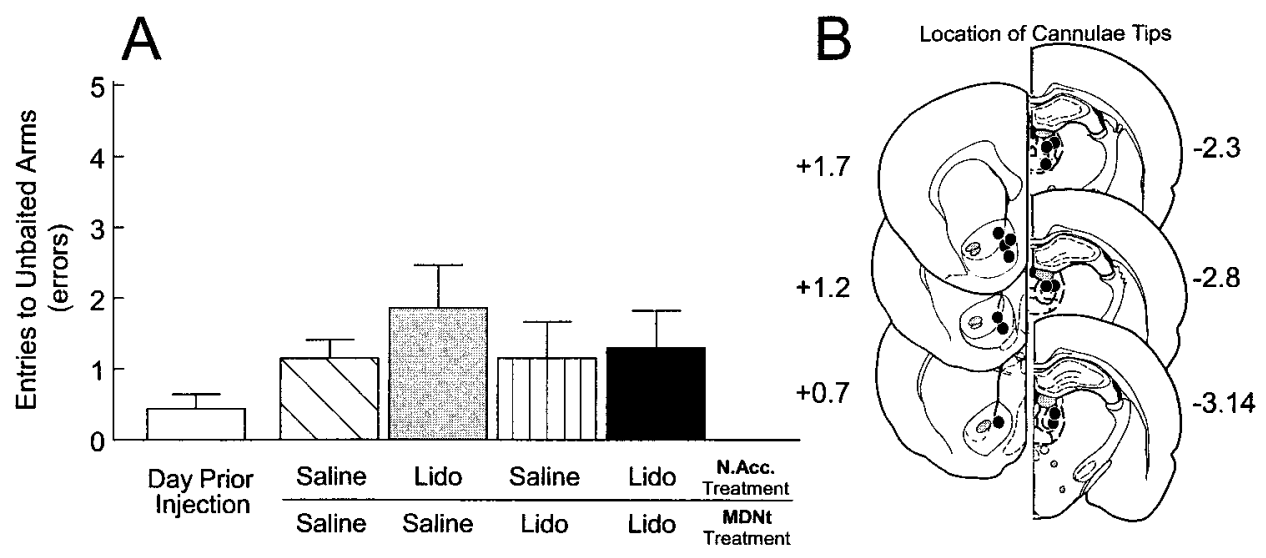

delayed SWSh task on 4 separate days. The analysis of these data revealed no significant main effect of treatment $\left(F_{(4,24)}=1.31\right.$; not significant; Fig. $3 A)$. There was a significant main effect of error type $\left(F_{(1,6)}=103.89 ; p<0.001\right)$. There was no significant treatment $\times$ error type interaction $\left(F_{(4,24)}=1.25\right.$; not significant).

Histology. The location of cannulae tips for all rats receiving MDNt-N.Acc. disconnections are represented in Figure $3 B$. Bilateral placements in the MDNt were similar to those observed in Experiment 1, whereas placements in the N.Acc. were located primarily in the medial shell region, similar to placements observed by Floresco et al. (1997).

\section{PFC-N.Acc. disconnections}

A separate group of rats $(n=8)$ with two sets of bilateral cannulae implanted into the prelimbic region of the PFC and the N.Acc. received the injection protocol described above before the test phase of the delayed SWSh task on 4 separate days. An analysis of these data revealed a significant main effect of treatment $\left(F_{(4,28)}=10.85 ; p<0.001\right)$. Tukey's post hoc test for repeated measures revealed that rats made significantly more errors on injection days on which the PFC and the N.Acc. both received lidocaine infusions and were disconnected versus all other injection days ( $p<0.01$; Fig. $4 A)$. There were no significant differences in the number of errors made on any of the other injection days. There was a significant main effect of error type $\left(F_{(1,7)}=25.84 ; p<0.001\right)$. Subsequent planned comparisons on the type of errors made after PFC-N.Acc. disconnections showed that rats made an equivalent number of across- and within-phase errors $\left(F_{(1,7)}=0.33\right.$; not significant; Fig. $4 A$, inset $)$. There was no significant treatment $\times$ error type interaction $\left(F_{(4,28)}=2.30\right.$; not significant). A separate series of tests was conducted to assess any hemispheric biases on the number of errors made after unilateral PFC inactivations, unilateral N.Acc. inactivations, and the disconnection lesions. The analysis revealed no significant effects of the side of the injection on the number of errors made by rats on the 3 injection days (all $F<0.5$; not significant).

Histology. The location of cannulae tips for all rats receiving PFC-N.Acc. disconnections are represented in Figure 4B. Bilateral placements in the prelimbic region of the $\mathrm{PFC}$ were similar to those observed after PFC-MDNt disconnections, whereas placements in the N.Acc. were located primarily in the medial shell region, similar to placements observed in rats receiving MDNtN.Acc. disconnections.

Latencies. Analysis of the latency data for all three groups in Experiment 2 revealed that there were no significant differences between treatment conditions for either the time to enter the first
Figure 4. Effects of PFC-N.Acc. disconnections on performance of the delayed SWSh task. $A$, Number of errors (mean \pm SEM) made by rats on the day before the first injection (open bar) and after unilateral inf usions of saline into both the PFC and the N.Acc. (hatched bar), unilateral infusions of lidocaine (Lido) into the PFC and contralateral saline in the N.Acc. ( gray bar), unilateral infusions of Lido into the N.Acc. and contralateral saline into the PFC (striped bar), and unilateral Lido into the N.Acc. and contralateral Lido into the PFC (disconnection; black bar) before the test phase of the delayed SWSh task. ${ }^{\star \star}$ Significance at $p<$ 0.001 versus all other treatment conditions. Inset, Number of across-phase (cross-hatched bar) versus within-phase (striped bar) errors made by rats during Lido/Lido (disconnection) injection days. B, Location of cannulae tips (black circles) for all rats used for data analysis receiving PFC-N.Acc. disconnections before the

delayed SWSh task. Plates are computer generated adaptations from Paxinos and Watson (1986). Numbers beside each plate correspond to millimeters from bregma.

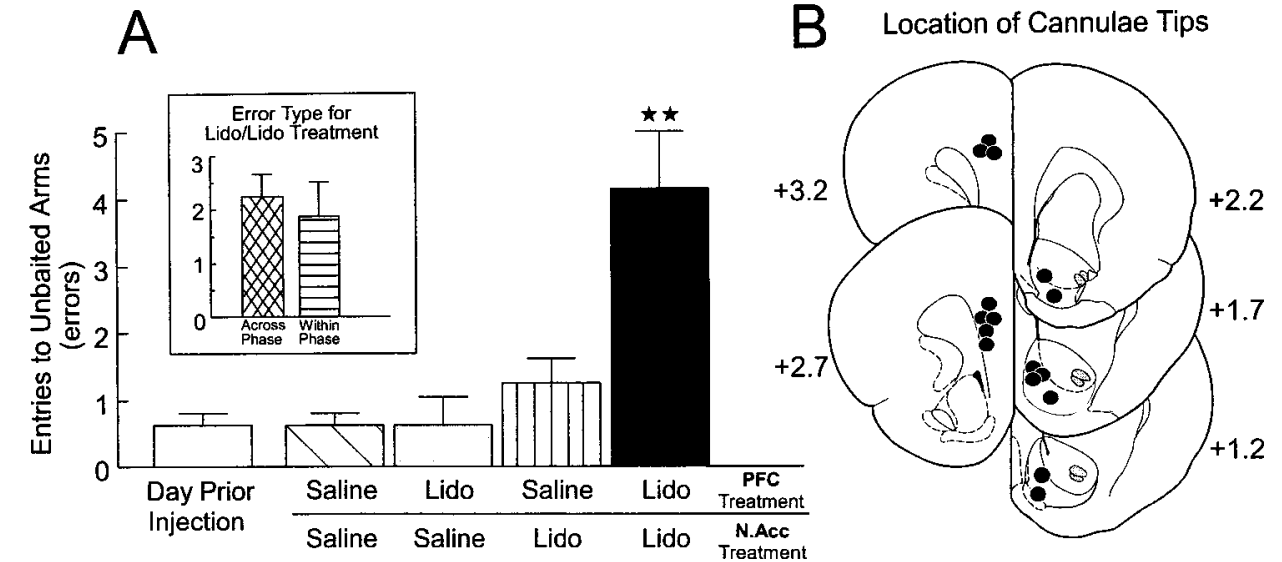

B Location of Cannulae Tips 
A

PFC-MDNt Disconnections

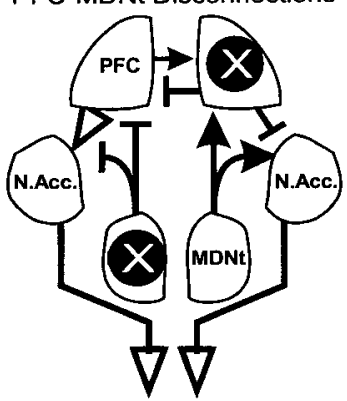

Impaired Behavior
B
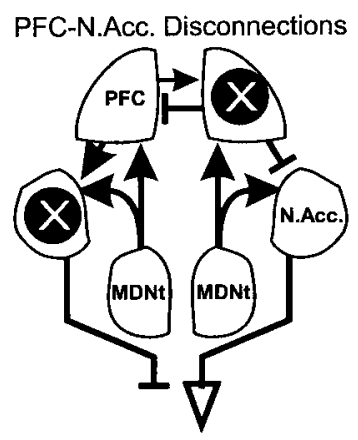

Impaired Behavior
C

MDNt-N.Acc. Disconnections

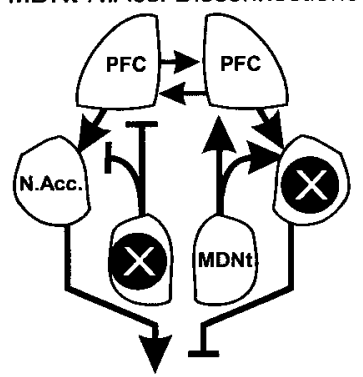

Unimpaired Behavior

Figure 5. Diagram of the anatomical connections investigated in the present study between the MDNt, the prelimbic cortex region of the PFC and the N.Acc. $X$, Location of the unilateral inactivations to the MDNt, PFC, or N.Acc. for the delayed SWSh task; solid arrows, intact pathways; open arrows, pathways that are not blocked but do not carry the relevant information because of a concomitant lidocaine-induced lesion upstream of this pathway; $T$ symbols, blocked, nonfunctional pathways. $A$, Proposed route of information transfer between the PFC and MDNt during the delayed SWSh task. By disconnecting the PFC-MDNt pathway, information cannot be processed by the PFC to generate appropriate responses after a delay, thereby disrupting appropriate output (impairment). B, Proposed route of information transfer between the PFC and N.Acc. during the delayed SWSh task. Disconnection of the PFC-N.Acc. pathway prevents the flow of information from the PFC through the N.Acc. to motor output centers (impairment). $C$, Proposed route of information transfer between the MDNt and N.Acc. during the delayed SWSh task. Information from the MDNt may be routed primarily through the PFC and subsequently to the N.Acc. Thus, although the pathway from the MDNt to the N.Acc. is disconnected, relevant information may still be transferred from the unanesthetized MDNt to the ipsilateral PFC and subsequently routed to the contralateral N.Acc., allowing for appropriate output (no impairment).

arm or on the average time per subsequent choice (all $F<1.6$; not significant).

\section{Discussion}

Lidocaine infusions in an asymmetrical pattern, which disconnected the flow of information between the PFC and the MDNt, disrupted the correct choice of arms containing food when administered before the test phase of the delayed SWSh task (Fig. $5 A$ ). These data suggest that intact serial transmission in this corticothalamic pathway is essential for performance of working memory tasks with extended delays. Analysis of the type of errors revealed that rats made more across-phase errors than withinphase errors. This result was unexpected, because bilateral inactivations of either structure alone (Seamans et al., 1995; Experiment 1) caused an equal number of both types of errors, a pattern that has been interpreted as a disruption in planning a foraging strategy, as opposed to a memory deficit per se. One possible explanation of increased visits to arms baited in the training session may be that disconnections between the PFC and the MDNt caused an impairment in behavioral flexibility (Hunt and Aggleton, 1998), resulting in preseveration. However, this explanation seems unlikely, given that bilateral inactivation of either the MDNt (Experiment 1) or the prelimbic region of the PFC (Seamans et al., 1995) did not result in preseverative responding on the delayed SWSh task. A more likely explanation for the selective increase in across-phase errors is that this neural circuit is specifically involved in the retrieval and use of trial-unique information acquired before a delay to guide choice behavior toward the probable location of food during the test phase. The finding that PFC-MDNt disconnections did not significantly disrupt within-phase performance indicates that this pathway does not play a role in monitoring choice behavior within a trial. The finding that bilateral inactivations of the MDNt disrupted withinphase performance, whereas PFC-MDNt disconnections did not, suggests that another population of MDNt neurons that does not project to the PFC may mediate within-phase performance. Support for this idea comes from the finding that a subset of MDNt neurons show selective increases in activity when an animal is presented with a conditional stimulus that requires the animal to inhibit an instrumental response (Kubota et al., 1996). Thus it may be that other efferent projections of the MDNt, such as to the orbitofrontal cortex or the hippocampal formation, are involved in monitoring responses within a trial. (Su and Bentivoglio, 1990; Ray and Price, 1992; Floresco et al., 1997; Rolls, 1998).

It is notable that the MDNt can be subdivided into separate regions based on cytoarchitectural properties and connectivity to cortical and subcortical regions (Groenewegen, 1988). Specifically, the medial segments of the MDNt receive projections from a number of limbic regions (e.g., amygdala and entorhinal cortex), whereas the central segments receive a more dense input from olfactory-related structures (Groenewegen, 1988). Similarly, the lateral segments of the MDNt send projections to the dorsal anterior cingulate cortex, whereas the medial segments send afferents to the prelimbic and infralimbic regions of the PFC (Conde et al., 1995). With respect to the present study, infusions of lidocaine into the MDNt would be expected to inactivate multiple subregions of this nucleus, including the central, lateral, and medial segments. Thus, it is difficult to determine the functional roles that each of these subnuclei play during the delayed SWSh task. However, in light of the results of Experiment 2, in which disconnections between the MDNt and the prelimbic region of the PFC disrupted delayed SWSh performance, it is reasonable to speculate that the more medial components of the MDNt (which project to the prelimbic and infralimbic areas) may play an important role in this form of working memory. More research is needed to ascertain the precise role that each of these thalamic subregions plays in working memory processes.

Disconnection of the projection between the PFC and the N.Acc. also disrupted performance on the delayed SWSh task. Rats made an equal number of across- and within-phase errors, which may be interpreted as a complete disruption of a prospective foraging strategy. These data confirm our previous hypothesis (Floresco et al., 1997) that inputs from the PFC to the N.Acc. are essential for the transformation of a prospective plan of action into appropriate behavioral output. Furthermore, these data are consistent with the more general theory that executive control over motor functions requires interactions between the PFC and striatal systems (Robbins, 1990, 1991; Goldman-Rakic et al., 1992). Thus, a unilateral inactivation of the PFC could deprive the N.Acc. in one hemisphere of information regarding a prospective foraging strategy, whereas direct inactivation of the 
contralateral N.Acc. blocks the integration of this information into accurate goal-directed responses, thereby leading to random responding and impaired foraging behavior (Fig. $5 B$ ).

In contrast to the above-mentioned findings, disconnections between the MDNt and the N.Acc. had no disruptive effect on delayed SWSh performance. This finding may seem contradictory, because bilateral inactivations of either the MDNt (Experiment 1) or the N.Acc. (Seamans and Phillips, 1994) impaired foraging on the delayed task. However, we have observed a similar pattern of results after disconnections between the ventral CA1-subiculum and the N.Acc. (Floresco et al., 1997). In that study, bilateral inactivations of the ventral subiculum disrupted delayed SWSh performance, whereas disconnections between the ventral subiculum and the N.Acc. did not. It was conjectured that during the delayed SWSh task, information was transferred serially from the hippocampus initially to the PFC and then to the N.Acc. Given the multiple efferent projections from the hippocampus, asymmetrical disconnections between the hippocampus and the N.Acc. would not disrupt foraging behavior, because information from the intact PFC would still have access to motor systems through the N.Acc via contralateral corticostriatal projections (Sesack et al., 1989; Brog et al., 1993; Conde et al., 1995). A similar mechanism may explain the lack of effect of MDNtN.Acc. disconnections in the present study. Information arising from the MDNt in the intact hemisphere would still be able to access the PFC, and in turn, outputs from the PFC would be able to interact with the intact N.Acc. in the opposite hemisphere via its contralateral connections (Fig. 5C). Thus, the present results in combination with our previous findings (Floresco et al., 1997) suggest that serial inputs from both the MDNt and the ventral subiculum converge in the PFC, and the resulting information is subsequently transferred to the N.Acc.

\section{GENERAL DISCUSSION}

The present data demonstrate a critical role for the MDNt in the performance of working memory tasks with extended delays. In Experiment 1, bilateral transient lesions of the MDNt selectively disrupted performance when administered before the test phase of the delayed SWSh task. Similar treatments had no effect when administered before the training phase or when administered before a nondelayed RF trial. Experiment 2 used asymmetrical disconnection lesions to assess the routes of serial transmission among the MDNt, the PFC, and the N.Acc. during the delayed SWSh task. The logic underlying the use of disconnection lesions to identify components of a functional neural circuit is based on the assumption that information is transferred serially from one structure to an efferent region on both sides of the brain in parallel. Furthermore, the design assumes that dysfunction will result from blockade of neural activity at the origin of a pathway in one hemisphere and the termination of the efferent pathway in the contralateral hemisphere. It follows that a unilateral inactivation at either site should have no effect on behavior. Using this procedure, it was revealed that performance on the delayed SWSh task is dependent on an intact neural circuit linking the MDNt to the PFC and the PFC to the N.Acc.

\section{A distributed circuit for working memory}

Previous research in our laboratory has examined the importance of interactions between the PFC and its subcortical afferents during working memory with extended delays. Specifically, we have shown that performance of the delayed SWSh task is critically dependent on a distributed cortical-subcortical network linking the PFC and the ventral subiculum (Floresco et al., 1997), suggesting that information maintained in a spatial memory buffer is routed from the hippocampus to the PFC, where it may be integrated into a prospective foraging strategy. Furthermore, we posited that "interactions between the PFC and the ventral striatum are involved in the transformation of spatial memory, processed by hippocampal-cortical circuits, into an efficient sequence of goal-directed motor responses" (Floresco et al., 1997, p 1889). This hypothesis was confirmed by the present finding that disconnections between the PFC and the N.Acc. disrupted delayed SWSh performance in a manner similar to PFC-hippocampal disconnections. We have also shown the importance of the mesocortical dopaminergic projection from the ventral tegmental area to the PFC in this type of working memory. Infusions of a D1 receptor antagonist bilaterally or asymmetrical infusions of a D1 antagonist into the PFC combined with an inactivation of the ventral subiculum disrupted performance on the delayed SWSh task (Seamans et al., 1998). These results suggested that PFC dopamine transmission plays an important neurmodulatory or "gain-amplifying" role (Robbins and Everitt, 1992), and may enhance task-relevant hippocampal inputs to the PFC.

Evidence from both clinical and preclinical studies suggests that the PFC interacts with the MDNt during certain types of learning and memory. Humans with thalamic damage show frontal-type deficits on a variety of neuropsychiatric tasks (Bogousslavsky et al., 1988; Joyce and Robbins, 1991; Daum and Ackermann, 1994). Likewise, it is well established that animals with experimentally induced lesions of the MDNt show behavioral deficits resembling those observed after PFC damage (Goldman et al., 1971; Isseroff et al., 1982; Gabriel, 1993; Harrison and Mair, 1996; Dias and Aggleton, 1997; Hunt and Aggleton, 1998). The present findings are consistent with the hypothesis that an interaction between the MDNt and the PFC forms an important neural component of executive processing in which previously acquired trial-unique information must be used to guide memorybased behavior after a delay. Disconnections between the PFC and the MDNt disrupted foraging during the test phase of the delayed SWSh task, causing a selective increase in across-phase errors. This pattern of errors suggests that this thalamocortical pathway is selectively involved in the retrieval of previously acquired information over a delay but not for the monitoring of choices within a trial. This result, in addition to our previous findings (Floresco et al., 1997), is consistent with the theoretical model of discriminative learning proposed by Gabriel (1990, 1993) and Freeman et al. (1996), which posits that topographically organized patterns of neural activity, spanning across a distributed neural circuit connecting the hippocampus, the MDNt, and cingulate cortex, mediates "working memory involved with context-based retrieval for relatively brief time intervals (minutes to hours)" (Freeman et al., 1996, p 1548). With respect to the delayed SWSh task, it is apparent that the synchronous convergence to the PFC of inputs originating in the hippocampus, the ventral tegmental area, and the MDNt is essential for the contextdependent retrieval and manipulation of recently acquired information. Subsequently, this information is integrated into a prospective foraging strategy that guides the animals toward arms that are predicted to contain food. The transformation of this strategy into behavioral output is mediated by an interaction between the PFC and the N.Acc. Furthermore, recent evidence suggests that the ventral striatum can interact with the ventral pallidum (Floresco et al., 1999) and the pedunculopontine nuclei 


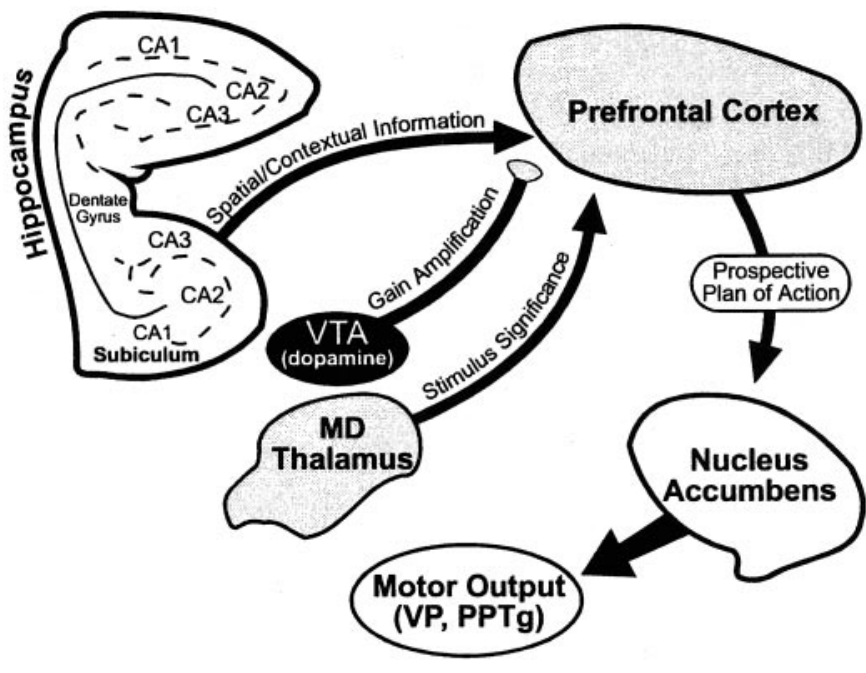

Figure 6. Summary diagram of the neural regions involved in delayed SWSh behavior. See General Discussion for details. VTA, Ventral tegmental area; $M D$ Thalamus, mediodorsal thalamus; $V P$, ventral pallidum; $P P T g$, pedunculopontine nuclei.

(Winn and Keating, 1998), forming a subsequent stage of limbicmotor integration (Fig. 6).

Further insight into the specific contribution of the MDNt to the executive processes that are engaged during the delayed SWSh task comes from electrophysiological recording studies in awake rodents. During conditional discrimination tasks, neurons in the MDNt or the PFC show a selective increase in activity when the animal is presented with a simple or configural conditional stimulus that is predictive of a motivationally significant event (e.g., shock or reinforcement) (Gabriel, 1990, 1993; Freeman et al., 1996; Oyoshi et al., 1996). These selective increases in neural activity by MDNt neurons suggest that these cells encode the motivational significance of particular stimuli in an environment. Moreover, these increases in neural activity occur shortly before an appropriate motor response is initiated. The increase in discriminatory neural activity in the MDNt precedes that observed in the PFC, and lesions to the MDNt abolish increased activity in PFC neurons (Gabriel, 1990), suggesting that information is transferred from the MDNt to the PFC when an animal discriminates between various stimuli. A neural system such as this, which is activated selectively when an animal is presented with stimuli that are predictive of forthcoming motivationally relevant events, is similar to the proposed "selective attention" module of the central executive (Baddeley, 1998). A selective attention mechanism, mediated by thalamocortical circuits, working in concert with hippocampal inputs that provide spatial and contextual information to the PFC, would facilitate the recognition of particular stimuli that have motivational significance. This concept is consistent with the theoretical framework of Fuster (1997), in which the presentation of specific external stimuli that are signals for prospective action could activate a distributed cortical and subcortical network (including the PFC, hippocampus, and MDNt), which represents that action, and then prepare the motor systems (including the ventral striatum) for the appropriate response. In the context of the present study, asymmetrical lesions would prevent the transfer of information concerning the "stimulus significance" (Oyoshi et al., 1996) of individual arms on the maze from the MDNt to the PFC, thereby impairing the rat's ability to attend to and subsequently enter arms that are predicted to contain food (Fig. 6).

\section{Different neural circuits mediate different forms of working memory}

It is becoming increasingly apparent that working memory is not a unitary phenomenon but rather a collection of distinct yet interrelated cognitive processes that facilitate the transformation of memory into action (Baddeley and Della Sala, 1996; Fuster, 1997; Goldman-Rakic, 1998; Callicott et al., 1999). It follows therefore that these different types of working memory are mediated by distinct neural circuits. One subtype of working memory discussed frequently in the rodent literature, derived from the original concept of Honig (1978) and developed further by Olton and Papas (1979), refers to the use of a simple retrospective strategy that can be used for the "remembrance of places past" (Olton and Samuelson, 1976). It is well established that this form of spatial working memory is mediated primarily by the hippocampal formation (Olton and Papas, 1979; Packard et al., 1989; Floresco et al., 1997) and not the PFC or the MDNt. A more complex form of working memory, discussed more frequently in primate and human studies, refers to the storage, manipulation, and use of recently acquired information to guide prospective action. These processes are more akin to the models of working memory put forth by Baddeley (1998), Fuster (1997), and Goldman-Rakic (1998), in which a central executive manipulates information stored in a short-term memory buffer to guide action. This is similar to the "working-with-memory" concept of Winocur (1992). This form of working memory is essential for cognitive processes used during delayed response tasks, including the delayed SWSh task used in the present study, and is subserved by a more complex prefrontal cortical neural network linked to hippocampus and the MDNt. It is important to note that although these different types of working memory use different neural circuits for the respective cognitive operations needed to solve problems requiring retrospective versus prospective strategies, both systems must interact with the same subcortical, striatal, pallidal, and mesencephallic output regions to transform these processes into behavioral responses (Seamans and Phillips, 1994; Floresco et al., 1997, 1999; Keating and Winn, 1998; Winn and Keating., 1998).

\section{REFERENCES}

Baddeley AD (1986) Working memory. Oxford: Clarendon.

Baddeley AD (1998) Recent developments in working memory. Curr Opin Neurobiol 8:234-238.

Baddeley A, Della Sala S (1996) Working memory and executive control. Philos Trans R Soc Lond B Biol Sci 351:1397-1404.

Batuev AS, Kurina NP, Shutov AP (1990) Unit activity of the medial wall of the frontal cortex during delayed performance in rats. Behav Brain Res 41:95-102.

Beckstead RM (1978) Afferent connections of the entorhinal area in the rat as demonstrated by retrograde cell-labeling with horseradish peroxidase. Brain Res 152:249-264.

Berendse HW, Groenewegen HJ (1990) Organization of the thalamostriatal projections in the rat, with special emphasis on the ventral striatum. J Comp Neurol 299:187-228.

Bogousslavsky J, Ferrazzini M, Regli F, Assal G, Tanabe H, DelaloyeBischof A (1988) Manic delirium and frontal-like syndrome with paramedian infarction of the right thalamus. J Neurol Neurosurg Psychiatry 51:116-119.

Brog JS, Salyapongse A, Deutch A, Zahm DS (1993) The pattern of afferent innervation of the core and shell in the "accumbens" part of the 
ventral striatum: immunohistochemical detection of retrogradely transported fluoro-gold. J Comp Neurol 338:255-278.

Callicott JH, Mattay VS, Bertolino A, Finn K, Coppola R, Frank JA, Goldberg TE, Weinberger DR (1999) Physiological characteristics of capacity constraints of working memory as revealed by functional MRI. Cereb Cortex 9:20-26.

Conde F, Maire-Lepoivre E, Audinat E, Crepel F (1995) Afferent connections of the medial frontal cortex of the rat. II. cortical and subcortical afferents. J Comp Neurol 325:567-593.

Cook RG, Brown RF, Riley DA (1985) Flexible memory processing by rats: use of prospective and retrospective information in the radial arm maze. J Exp Psychol Anim Behav Process 11:453-469.

Daum I, Ackermann H (1994) Frontal-type memory impairment with thalamic damage. Int J Neurosci 77:187-198.

Dias R, Aggleton JP (1997) A comparison of the effects of prelimbic and anterior cingulate cortex lesions in the rat. Soc Neurosci Abstr 23:1606.

Dunnett SB (1990) Role of the prefrontal cortex and striatal output systems in short-term memory deficits associated with aging, basal forebrain lesions, and cholinergic-rich grafts. Can J Psychiatry 44:210-232.

Floresco SB, Seamans JK, Phillips AG (1997) Selective roles for hippocampal prefrontal cortical, and ventral striatal circuits in radial-arm maze tasks with or without a delay. J Neurosci 17:1880-1890.

Floresco SB, Braaksma DN, Phillips AG (1999) Involvement of the ventral pallidum in working memory tasks with or without a delay. Ann NY Acad Sci 877:711-716.

Freeman JH, Cuppernell C, Flannery K, Gabriel M (1996) Contextspecific multi site cingulate cortical, limbic thalamic, and hippocampal neuronal activity during concurrent discriminative approach and avoidance training in rabbits. J Neurosci 16:1538-1549.

Fuster JM (1995) Memory in the cerebral cortex: an emperical approach to neural networks in the human and nonhuman primate. Cambridge, MA: MIT.

Fuster JM (1997) Network memory. Trends Neurosci 20:451-459.

Fuster JM, Bauer RH, Jervey JP (1985) Functional interactions between inferotemporal and prefrontal cortex in a cognitive task. Brain Res 330:299-307.

Gabriel M (1990) Functions of anterior and posterior cingulate cortex during avoidance learning in rabbits. In: Progress in brain research, Vol 85 (Uylings HBM, Van Eden CG, De Bruin JPC, Corner MA, Feeustra MGP, eds), pp 467-483. Amsterdam: Elsevier.

Gabriel M (1993) Discriminative avoidance learning: a model system. In: Neurobiology of the cingulate cortex and limbic thalamus: a comprehensive handbook (Vogt BA, Gabriel M, eds), pp.478-523. Boston: Birkhäuser.

Gallassi R, Morreale A, Montagna P, Gambetti P, Lugaressi E (1992) "Fatal familial insomnia": neuropsychological study of a disease with thalamic degeneration. Cortex 28:175-187.

Goldman PS, Rosvold HE, Vest B, Galkin TW (1971) Analysis of the delayed-alternation deficit produced by dorsolateral prefrontal lesions in the rhesus monkey. J Comp Physiol Psychol 77:212-220.

Goldman-Rakic PS (1987) Circuitry of the prefrontal cortex and its regulation of behavior by representational knowledge. In: Handbook of physiology, Vol 5 (Mountcastle PF, ed), pp 373-417. Bethesda, MD: American Physiological Association.

Goldman-Rakic PS (1995) Cellular basis of working memory. Neuron 14:477-485.

Goldman-Rakic PS (1998) The prefrontal landscape: implications of functional architecture for understanding human mentation and the central executive. In: The prefrontal cortex: executive and cognitive functions (Roberts AC, Robbins TW, Weizkrantz L, eds), pp 67-86. Oxford: Oxford UP.

Goldman-Rakic PS, Bates JF, Chafee MW (1992) The prefrontal cortex and internally generated motor acts. Curr Opin Neurobiol 2:803-835.

Granon S, Poucet B (1995) Medial prefrontal lesions in the rat and spatial navigation: evidence for impaired planning. Behav Neurosci 109:474-484.

Groenewegen HJ (1988) Organization of the afferent connections of the mediodorsal thalamic nucleus in the rat, related to the mediodorsalprefrontal topography. Neuroscience 24:379-341.

Gutnikov SA, Ma YY, Gaffan D (1997) Temporo-frontal disconnection impairs visual-visual paired association learning but not configural learning in Macaca monkeys. Eur J Neurosci 9:1524-1529.

Harrison LM, Mair RG (1996) A comparison of the effects of frontal cortical and thalamic lesions on measures of spatial learning and memory in the rat. Behav Brain Res 75:195-206.

Honig WK (1978) Working memory in the pigeon. In: Cognitive aspects of animal behavior (Hulse SH, Honig WK, Fowler H, eds), pp 211-248. Hillsdale, NJ: Erlbaum.

Hunt PR, Aggleton JP (1998) Neurotoxic lesions of the dorsomedial thalamus impair the acquisition but not the performance of delayed matching to place by rats: a deficit in shifting response rules. J Neurosci 18:10045-10052.

Isseroff A, Rosvold HE, Galkin TW, Goldman-Rakic PS (1982) Spatial memory impairments following damage to the mediodorsal nucleus of the thalamus in rhesus monkeys. Brain Res 232:97-113.

Joyce EM, Robbins TW (1991) Frontal lobe function in Korsakoff and non-Korsakoff alcoholics: planning and spatial working memory. Neuropsychologia 29:709-723.

Keating GL, Winn P (1998) Radial arm maze performance after excitotoxiclesions of the pedunculopontine tegmental nucleus in rats. I. The random foraging task. Soc Neurosci Abstr 24:175.

Kessler J, Markowitsch HJ, Otto B (1982) Subtle but distinct impairments of rats with chemical lesions in the thalamic mediodorsal nucleus, tested in a radial-arm maze. J Comp Physiol Psychol 96:712-720.

Kimberg DY, D'Esposito M, Farah MJ (1997) Cognitive functions in the prefrontal cortex-working memory and executive control. Curr Dir Psychol Sci 6:185-192.

Kolb B, Pittman K, Sutherland RJ, Wishaw IQ (1982) Dissociation of the contributions of the prefrontal cortex and dorsomedial thalamic nucleus to spatially guided behavior in the rat. Behav Brain Res 6:365-378.

Krettek JE, Price J (1977) The cortical projections of the mediodorsal nucleus and adjacent thalamic nuclei in the rat. J Comp Neurol 171:157-192.

Kubota Y, Wolske M, Poremba A, Kang E, Gabriel M (1996) Stimulusrelated and movement-related single-unit activity in rabbit cingulate cortex and limbic thalamus during performance of discriminative avoidance behavior. Brain Res 721:22-38.

Leonard CM (1969) The prefrontal cortex of the rat. I. cortical projections of the mediodorsal nucleus. II. efferent connections. Brain Res 12:321-343.

Mair RD, Burk JA, Porter MC (1998) Lesions of the frontal cortex, hippocampus, and intralamina thalamic nuclei have distinct effects on remembering in rats. Behav Neurosci 112:772-792.

Mumby DG, Pinel JPJ, Dastur FN (1993) Mediodorsal thalamic lesions and object recognition in rats. Psychobiology 21:27-36.

Olton DS, Papas BC (1979) Spatial memory and hippocampal function. Neuropsychologia 17:669-682.

Olton DS, Samuelson RJ (1976) Remembrance of places past: spatial memory in rats. J Exp Psychol Anim Behav Process 2:97-116.

Orlov AA, Kurzina NP, Shutov AP (1988) Activity of medial wall neurons in frontal cortex in rat brains during delayed response reactions. Neurosci Behav Physiol 18:31-37.

Otake K, Nakamura Y (1998) Single midline thalamic neurons projecting to both the ventral striatum and the prefrontal cortex in the rat. Neuroscience 86:635-649.

Oyoshi T, Nishijo H, Asakura T, Takamura Y, Ono T (1996) Emotional and behavioral correlates of mediodorsal thalamic neurons during associative learning in rats. J Neurosci 16:5812-5829.

Packard MG, Hirsh R, White NM (1989) Differential effects of fornix and caudate nucleus lesions on two radial arm maze tasks: evidence for multiple memory systems. J Neurosci 9:1465-1472.

Packard MG, Regenold W, Quirion R, White NM (1990) Post-training injection of the acetylcholine M2 receptor antagonist AF-DX 116 improves memory. Brain Res 524:72-76.

Paxinos G, Watson C (1986) The rat brain in stereotaxic coordinates, Ed 2. New York: Academic.

Quintana J, Fuster JM, Yajeya J (1989) Effects of cooling parietal cortex on prefrontal units on delayed tasks. Brain Res 503:100-110.

Ragozzino ME, Detrick S, Kesner RP (1999) Involvement of the prelimbic-infralimbic areas of the rodent prefrontal cortex in behavioral flexibility for place and response learning. J Neurosci 19:4585-4594.

Rainer G, Rao SC, Miller EK (1999) Prospective coding for objects in primate prefrontal cortex. J Neurosci 19:5493-5505.

Ray JP, Price JL (1992) The organiztion of the thalamocortical connections of the mediodorsal thalamic nucleus in the rat, related to the ventral forebrain-prefrontal cortex topography. J Comp Neurol 323:167-197. 
Robbins TW (1990) The case for frontalstriatal dysfunction in schizophrenia. Schizophrenia Bull 16:391-402.

Robbins TW (1991) Cognitive deficits in schizophrenia and Parkinson's disease: neural basis and the role of dopamine. In: The mesolimbic dopamine system: from motivation to action (Willner P, Scheel-Kruger J, eds), pp 497-528. New York: Wiley.

Robbins TW, Everitt BJ (1992) Functions of dopamine in the dorsal and ventral striatum. Semin Neurosci 4:119-127.

Rolls ET (1998) The orbitofrontal cortex. In: The prefrontal cortex: executive and cognitive functions (Roberts AC, Robbins TW, Weizkrantz L, eds), pp 67-86. Oxford: Oxford UP.

Savage LM, Sweet AJ, Castillo R, Langlais PJ (1997) The effects of lesions to thalamic lateral internal medullary lamina and posterior nuclei on learning, memory and habituation in the rats. Behav Brain Res 82:133-147.

Seamans JK, Phillips AG (1994) Selective memory impairments produced by transient lidocaine-induced lesions of the nucleus accumbens in rats. Behav Neurosci 108:456-468.

Seamans JK, Floresco SB, Phillips AG (1995) Functional differences between the prelimbic and anterior cingulate regions of rat prefrontal cortex. Behav Neurosci 109:1063-1073.

Seamans JK, Floresco SB, Phillips AG (1998) $D_{1}$ receptor modulation of hippocampal-prefrontal cortical circuits integrating spatial memory with executive functions in the rat. J Neurosci 18:1613-1621.

Sesack SR, Deutch AY, Roth RH, Bunney BS (1989) Topographical organization of the efferent projections of the medial prefrontal cortex in the rat: an anterograde tract-tracing study with Phaseolus vulgaris leucoagglutinin. J Comp Neurol 290:213-242.

Shallice T (1982) Specific impairments of planning. Philos Trans R Soc Lond B Biol Sci 298:199-209.

Shallice T, Burgess P (1996) The domain of supervisory processes and temporal organization of behaviour. Philos Trans R Soc Lond B Biol Sci 351:1405-1411.

Stokes KA, Best PJ (1990) Mediodorsal thalamus lesions in rats impair radial-arm maze performance in a cued environment. Psychobiology 18:63-67.

Su HS, Bentivoglio M (1990) Thalamic midline cell populations projecting to the nucleus accumbens, amygdala, and hippocampus in the rat. J Comp Neurol 297:582-593.

Tehovnik EJ, Sommer MA (1997) Effective spread and timecourse of neural inactivation caused by lidocaine injection in monkey cerebral cortex. J Neurosci Methods 74:17-26.

Winn P and Keating GL (1998) Radial arm maze performance after excitotoxic lesions of the pedunculopontine tegmental nucleus in rats. II. The delayed spatial win shift task. Soc Neurosci Abstr 24:175.

Winocur G (1985) The hippocampus and thalamus: their roles in shortand long-term memory and the effects of interference. Behav Brain Res $16: 135-152$.

Winocur G (1992) A comparison of normal old rats and young adult rats with lesions to the hippocampus or prefrontal cortex on a test of matching-to-sample. Neuropsychologia 30:769-781. 